\title{
How dominant alleles influence progress toward speciation by natural selection
}

\author{
Aslı D. Munzur ${ }^{1, \bowtie}$ and Ken A. Thompson ${ }^{1, \infty}$ \\ ${ }^{1}$ Department of Zoology \& Biodiversity Research Centre, University of British Columbia, Canada
}

\begin{abstract}
Extrinsic post-zygotic isolating barriers occur when hybrids have poor fitness in the parental habitat(s) and ultimately arise as a consequence of interactions between the alleles underlying adaptation. Most models of speciation via natural selection consider only alleles that have additive phenotypic effects, even though many quantitative trait loci exhibit some dominance. We used computer simulations to evaluate how hybrid fitness differs when parent populations adapt from either (i) $\mathrm{mu}$ tations that are strictly additive or (ii) mutations with a range of dominance coefficients. Compared to the additive case, we find that introducing dominance invariably reduces hybrid fitness when parents adapt to identical optima (i.e., parallel selection) because hybrids express transgressive phenotypes that are maladaptive in the common parental niche. The effect of dominance becomes less negative as selection tends toward completely divergent. When selection is completely divergent, dominance can increase hybrid fitness over the additive case because chance events result in unequal mean dominance among populations and cause hybrids to resemble one of the parents rather than a maladapted intermediate phenotype. However, when the adapting population has many traits (i.e., high 'dimensionality') dominance always reduces hybrid fitness over the additive case because hybrids express maladaptive transgressive phenotypes in traits under stabilizing selection. Our results indicate that dominant alleles facilitate progress toward speciation via parallel natural selection, and might either increase or decrease the efficacy of divergent selection for speciation.
\end{abstract}

dominance | hybridization | post-zygotic isolation | simulation | hybrid fitness Correspondence: admunzur.21@gmail.com; ken.thompson@zoology.ubc.ca

\section{Introduction}

Adaptation by natural selection is increasingly recognised as a major cause of speciation (Nosil, 2012; Rabosky et al., 2018; Schluter, 2000). Under speciation-by-selection, reproductive isolation is caused by properties of the alleles used for adaptation (Langerhans and Riesch 2013; Schluter 2009). For post-zygotic isolating barriers, which reduce the fitness of hybrids, reproductive isolation results as a consequence of previously allopatric alleles 'meeting' in hybrid genomes (Coyne and Orr 2004). Poor hybrid fitness allows species barriers to persist by reducing the ability of hybrids to backcross with parents (Irwin, 2020) and thereby prevents introgression and gene flow.

Dominant beneficial mutations, where the phenotype of a heterozygote is more similar to the derived homozygote than the ancestral (i.e., 'wild-type') homozygote, are more likely to fix than their recessive counterparts (Haldane
1924). In empirical systems, the alleles underlying quantitative traits, which were presumably used for adaptation, have an array of dominance coefficients (e.g., Miller et al. 2014). Moreover, a recent meta-analysis indicates that the traits of $F_{1}$ hybrids are typically more dominant than additive (Thompson et al., 2021). Yet, it is unclear how dominant alleles affect progress toward speciation by natural selection. Importantly, the effects of dominance on hybrid fitness are expected to change with the form of selection acting on populations- that is, the extent of (non)parallelism (Bolnick et al., 2018). When populations adapt to similar environments, empirical studies have shown that the alleles can be incompatible due to populations 'overshooting' the phenotypic optimum (Ono et al. 2017). When populations adapt in perpendicular directions, dominance might result in trait 'mismatches' that reduce hybrid fitness (Thompson et al., 2021). Whereas when populations adapt in opposite directions dominance might balance out in hybrids and have little effect on fitness.

In this study, we use simulations to provide novel insight into how non-additive trait expression affects progress toward speciation via reduced hybrid fitness. We investigate how the availability of dominant alleles for adaptation affects progress toward speciation via 'extrinsic' hybrid fitness and consider adaptation along a continuum of forms of natural selection from completely parallel to completely divergent. Defining parallel evolution as a spectrum rather than a binary concept is increasingly accepted in evolutionary biology (Bolnick et al. 2018; De Lisle and Bolnick 2020; James et al. 2020) and variation in phenotypic parallelism can be quantified empirically using approaches such as Phenotypic Vector Change Analysis (Adams and Collyer 2009; Oke et al. 2017; Stuart et al. 2017). We specifically compare hybrid fitness between simulations where all alleles are co-dominant (the scenario with no dominance) to simulations where alleles can have a range of dominance effects. In line with previous empirical work, we predicted that dominance would decrease hybrid fitness under highly parallel selection due to acquisition of transgressive phenotypes and have less of an effect as selection was less parallel. Overall, our results illustrate how a key feature of quantitative trait loci-the dominance coefficient-influences progress toward speciation by natural selection.

\section{Methods}

We used computer simulations to study the effect of dominant alleles on speciation-by-selection via the evolution of 
bioRxiv preprint doi: https://doi.org/10.1101/2021.06.30.450598; this version posted July 1, 2021. The copyright holder for this preprint (which was not certified by peer review) is the author/funder, who has granted bioRxiv a license to display the preprint in perpetuity. It is made available under aCC-BY-NC-ND 4.0 International license.
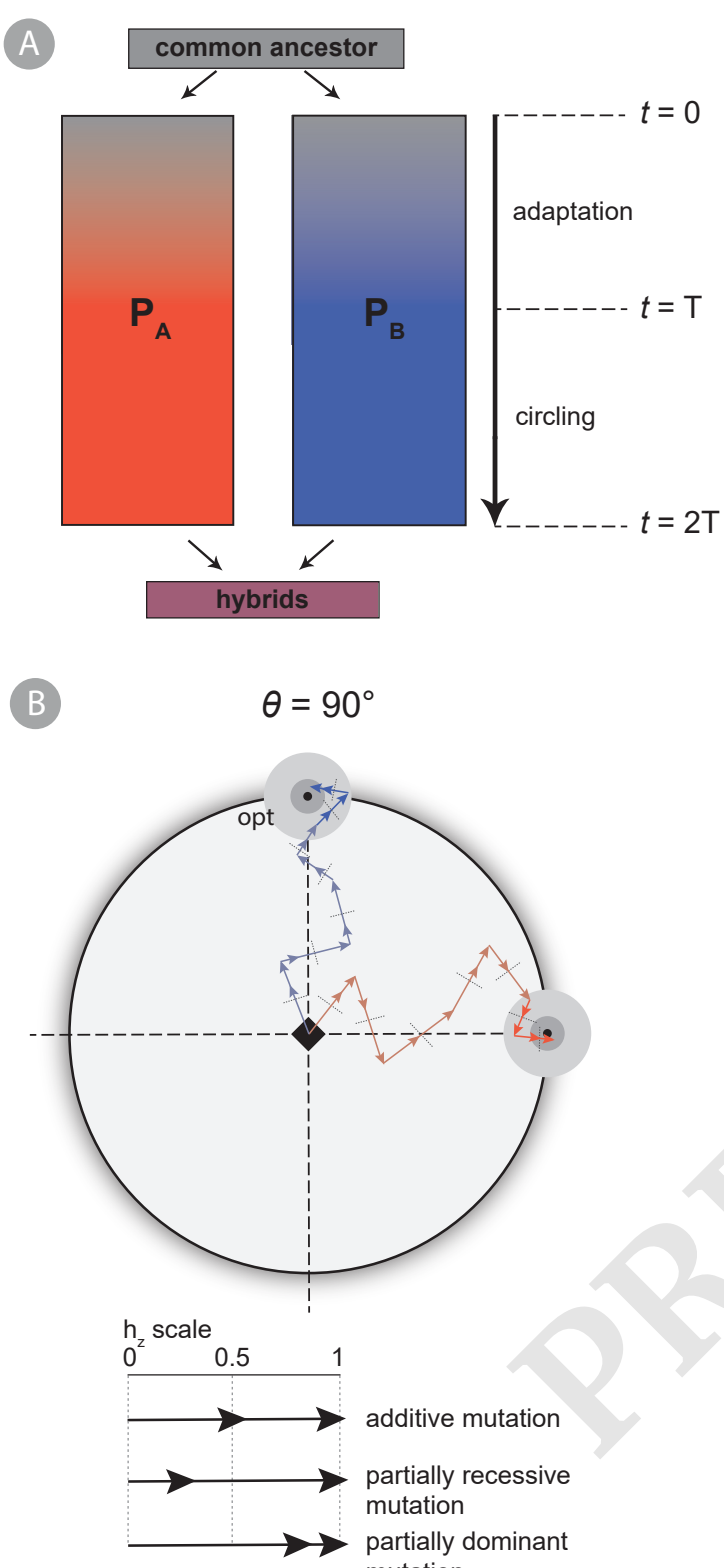

additive mutation

partially recessive mutation

partially dominant mutation

Fig. 1. Overview of simulation structure and concepts. Panel $(A)$ shows how the individual simulations were run. Two independent 'parental' populations, $P_{A}$ and $P_{B}$, first evolve in allopatry for $T$ generations, where a new mutation arises each generation during adaptation. Once the adaptation phase is complete, $T$ additional mutations arise during the circling phase. After $2 T$ total generations, the two populations interbreed to form hybrids. Panel (B) illustrates how the two populations adapt towards their respective phenotypic optima, and this case is showing an angle of divergence of $90^{\circ}$ (i.e., $\theta=90^{\circ}$ ). Contours indicate the phenotypic optima and the black diamond represents the origin. The arrows at the bottom of the panel illustrate three informative cases of phenotypic dominance for a mutation with a given effect size-additive $\left(h_{\mathrm{z}}=0.5\right)$; partially recessive $\left(h_{\mathrm{z}}<0.5\right)$; and partially dominant $\left(h_{z}>0.5\right)$. The leftmost arrowhead indicates the effect when heterozygous and the rightmost arrowhead indicates the effect when homozygous for the derived allele.
Table 1. Parameters used in the main text simulations (other values considered in supplement).

\begin{tabular}{ll}
\hline Parameter & Value \\
\hline$\alpha$, mutation effect size & $0.1 / \sqrt{n}$ \\
$d$, distance to phenotypic optima & 1 \\
$\sigma$, selection strength & 5 \\
$n$, dimensionality (\# traits) & 2 \\
$\theta$, angle of divergence & $0^{\circ} \leq \theta \leq 180^{\circ}$ \\
$h_{\mathrm{z}}$, phenotypic dominance coefficient & $0 \leq h_{\mathrm{z}} \leq 1$ (var. dom.); 0.5 (no dom.) \\
\hline
\end{tabular}

reduced hybrid fitness (i.e., post-zygotic isolation). The particular scenario considered by our simulations is modelled after that of several recent studies (Chevin et al., 2014; Thompson et al., 2019; Yamaguchi and Otto, 2020). Specifically, we model two parental populations that begin with the same ancestral phenotype and undergo adaptive divergence without gene flow (i.e., allopatry) toward their respective prespecified phenotypic optima (Fig. 1). After both populations reach their optima, they are given the opportunity to oscillate or 'circle' around it for a fixed duration that is the same length of the earlier 'adaptation' phase. The parental populations then hybridize to form early-generation hybrids $\left(\mathrm{F}_{1}\right.$, $\mathrm{F}_{2}$ and $\mathrm{BC}_{1}$ ). We compare the fitness of these hybrids among simulations runs under different conditions - the key manipulation being whether alleles have variable dominance or are all additive. We consider hybrid fitness as our main response variable and a proxy for the strength of post-zygotic reproductive isolation that separates the parents (Irwin, 2020).

Genetics and biology. We envision a scenario where two diploid populations with a single pair of chromosomes diverge from an ancestral population, colonize and adapt to two novel environments, then hybridize (Fig. 1). We use an infinite sites model (Kimura, 1969), where each mutation occurs at a unique locus and there is no linkage among loci (i.e., free recombination among loci). We assume that populations are effectively monomorphic such that all mutations reach fixation before subsequent mutations occur. Parental populations are initially identical, and adapt towards their respective optima by fixing mutations that modify their phenotypes. We assume individuals are obligatorily sexual and hermaphroditic.

Simulations occur within the framework of Fisher's (1930) geometric model. The phenotype of an individual, $\mathbf{z}$, is an $n$ dimensional vector, $\mathbf{z}=\left[z_{1}, z_{2}, \ldots, z_{n}\right]$, with $n$ being the effective number of traits or 'dimensionality' (Tenaillon 2014). The value of each phenotypic trait, $z_{i}$, is determined additively by summing the phenotypic effects of all mutations on trait $i$ held by an individual. Mutations arise in a single individual in a heterozygous state, and individual mutations are associated with a vector of mutational effects, $\Delta \boldsymbol{Z}$, that define the mutation's phenotypic effect on each trait when homozygous for the derived allele. Mutations can affect all traits (i.e., universal pleiotropy), where the effect on each trait is drawn from a random normal distribution with mean of 0 and standard deviation of $\alpha$. Specific parameters used in the simulations are summarized in Table 1. 
Fitness and mutation fixation. Fitness is a Gaussian function that depends the Euclidean distance between the individual's phenotype and the phenotypic optimum, $\|\mathbf{z}-\mathbf{o}\|$, and the strength of selection, $\sigma$ (Lande, 1979):

$$
W=\exp \left(-\sigma\|\mathbf{z}-\mathbf{o}\|^{2} / 2\right)
$$

For each newly arisen mutation, initially in the heterozygous state, we consider its dominance coefficient and selection coefficient to determine its fixation probability because we assume mutations either fix or are lost completely. To calculate the selection coefficient of a mutation $(s)$, we consider the fitness of both the mutant and wild-type phenotype :

$$
s \equiv \frac{w(\mathbf{Z}+\Delta \mathbf{Z})}{w(\mathbf{Z})}-1
$$

where $w(\mathbf{Z}+\Delta \mathbf{Z})$ is the fitness of the homozygous mutant and $w(\mathbf{Z})$ is the fitness of the wild-type.

We calculate the dominance for fitness of the allele, $h_{w}$ as:

$$
h_{w} \equiv\left(\frac{w\left(\mathbf{Z}+\Delta \mathbf{Z} h_{\mathrm{z}}\right)}{w(\mathbf{Z})}-1\right) / s,
$$

where $h_{z}$ is the phenotypic dominance of the mutation.

After a mutation arises, the probability it fixes is calculated according to the fixation probability equation (Haldane, 1924, 1927):

$$
\pi \equiv 2 h_{w} s
$$

where $s$ is the selection coefficient of the given mutation and $h_{w}$ is the dominance for the fitness of the derived allele, as calculated by Equation 3. We compared fixation probabilities from Eqn. 4 to probabilities calculated from equations with more parameters (e.g., considering $N_{e}$ ) and found they were highly correlated (Fig. S1). Thus, we used the simplest equation. Fixed mutations are incorporated into the population to influence the phenotype and thus comprise the wild-type in the subsequent generation.

Variation in dominance. Phenotypic dominance coefficients, $h_{z}$, are assigned to mutations when they arise and range uniformly between 0 and 1 (i.e., phenotypic under- and overdominance are impossible by assumption). Additive (i.e., codominant) mutations have $h_{z}=0.5$, whereas completely recessive and dominant mutations have values of $h_{z}=0$ and $h_{z}$ $=1$, respectively. We run individual simulations under two different conditions: (i) no dominance and (ii) variable dominance. Under 'no dominance', $h_{z}=0.5$ for all derived alleles. Under 'variable dominance', $h_{z}$ values for each mutation are drawn from a random uniform distribution where $0 \leq h_{z} \leq 1$.

Adaptation and circling phase. In each iteration of our simulations, two parental populations $\left(\mathrm{P}_{\mathrm{A}}\right.$ and $\left.\mathrm{P}_{\mathrm{B}}\right)$ begin at the origin, defined as $\mathbf{z}_{0}=[0,0, \ldots 0]$, and adapt to prespecified and fixed optima. During the adaptation phase, both parental populations encounter the same set of mutations in the same order, which allows us to isolate the role of selection when determining hybrid fitness under different assumptions about genetic parallelism (see below). This choice to have both populations encounter identical mutations does not affect our qualitative conclusions-simulations where $\mathrm{P}_{\mathrm{A}}$ and $P_{B}$ encountered unique mutations, or could fix identical alleles from standing variation (as in Thompson et al. (2019)) arrived at the same qualitative conclusions (not shown).

We divide the dynamics of evolution into two phases: adaptation and circling. Each run lasts a sufficient duration such that an equivalent number of mutations arise during the adaptation as in the circling phase (i.e., $2 T$ mutations arise during each iteration). We determine this by recording the point at which both populations have travelled $95 \%$ of the distance to their respective phenotypic optimum (i.e., $\|\mathbf{z}-\mathbf{o}\|<0.95 d$ ). When both populations reach to this point, another set of $T$ mutations arise until the simulation ends (Fig. 1).

Even though the parental populations encounter the same set of mutations in the same order, the distribution of the dominance coefficients $\left(h_{\mathrm{A}}\right.$ and $\left.h_{\mathrm{B}}\right)$ in their genomes can differ if different mutations are fixed. To quantify the distribution of the dominance coefficients across the two parental populations, we first calculate the mean dominance coefficients across parents, and then use these values to calculate the 'dominance imbalance' within a given replicate (simulation run) as follows:

$$
h_{\text {imbalance }} \equiv \frac{\left|\overline{h_{z \mathrm{~A}}}-\overline{h_{z \mathrm{~B}}}\right|}{n_{f}},
$$

where $\overline{h_{z \mathrm{~A}}}$ and $\overline{h_{z \mathrm{~B}}}$ are the mean dominance coefficients for the first and second parental populations, and $n_{f}$ stands for the total number of fixed mutations.

Phenotypic optima. In our simulations, both parental populations adapt to novel environments that are equidistant from the origin. The first parental population, $\mathrm{P}_{\mathrm{A}}$, always adapts to $\mathbf{o}_{\mathrm{P}_{\mathrm{A}}}=[d, 0,0, \ldots 0]$. The second population adapts to an optimum that has an equivalent vector length $(d)$ at a location defined by the parameter $\theta$, representing the angle of divergence between the two phenotypic optima. The concept of $\theta$ is further illustrated in Fig. 1B. We considered various values of $\theta$ that range from $0^{\circ}$, where both populations have the identical optima, to $180^{\circ}$, where populations adapt to optima in exact opposite directions from the origin and acquire opposite traits (i.e., $\mathbf{o}_{\mathrm{P}_{\mathrm{B}}}=[-d, 0, \ldots 0]$ ). For intermediate angles between $0^{\circ}$ and $180^{\circ}, \theta$ only affects the first two traits while the others remain under stabilizing selection at 0 .

Hybridization \& hybrid fitness. After both populations reach and circle their respective optima, they interbreed to form hybrids with free recombination across all loci. We form $\mathrm{F}_{1}, \mathrm{~F}_{2}$, and back-cross $\left(\mathrm{BC}_{1}\right)$ hybrids and measure their fitness under several different assumptions. $F_{1}$ hybrids are completely heterozygous across the genome and are all identical in terms of genotype and phenotype. For $F_{2}$ and backcross hybrids, $\mathrm{F}_{1}$ hybrids transmit either the $\mathrm{P}_{\mathrm{A}}$ or $\mathrm{P}_{\mathrm{B}}$ allele at each locus with equal probability. We allow hybrids 
to 'choose their environment' where we only consider the greater of the two mean fitness values for each individual measured in both parental environments. We then average across the individual hybrid fitness values to find the fitness of a hybrid class. We form $500 \mathrm{~F}_{2}$ and $500 \mathrm{BC}_{1}$ hybrids each and consider their mean fitness. Although we discuss results for all hybrid classes, we primarily focus on the fitness of $F_{1}$ hybrids because this is the most important stage for speciation.

Parallel genetic evolution. Populations evolving towards similar optima often fix the same alleles (Conte et al., 2012; Thompson et al., 2019). In our simulations, the two parental populations encounter mutations with identical phenotypic effects in the exact same order. We manipulate parallel evolution by changing our assumption of whether phenotypically identical alleles occur at different loci (parallel evolution impossible) or at the same locus (parallel evolution possible) in the two parental populations. Alleles that fix in both populations do not segregate (i.e., they are homozygous) in all hybrids. This assumption is biologically unrealistic in that the majority of parallelism is likely caused by the use of the same alleles from standing variation rather than parallel fixation of de novo mutations (but see Schluter 2009). However, the purpose of this manipulation is to provide a qualitative, rather than quantitative, understanding of the effect of parallel evolution on our conclusions. That is, we want to understand whether (effect or no effect) and how (positive or negative) parallelism affects the fitness of hybrids, not estimate its overall importance which is likely to vary by system (Conte et al., 2012).

To measure the extent of parallel evolution across parental populations, we considered the following equation (Thompson et al. 2019):

$$
P_{g}=\frac{1}{2}\left(\frac{f_{\mathrm{A}, \mathrm{B}}}{f_{\mathrm{A}}}+\frac{f_{\mathrm{A}, \mathrm{B}}}{f_{\mathrm{B}}}\right)
$$

where $f_{\mathrm{A}, \mathrm{B}}$ represents the number of alleles that fixed at the same locus in both parental populations, and $f_{\mathrm{A}}$ and $f_{\mathrm{B}}$ represent the alleles that fixed only in one population. $P_{g}$ ranges between 0 and 1; values closer to 1 indicate an increasing number identical alleles fixed in both populations. $P_{g}=0$ by definition is in simulation runs without parallel evolution.

\section{Results and discussion}

Effects of dominance on hybrid fitness. We considered the effect of variable dominance on the fitness of all classes of hybrids, but largely focus on $\mathrm{F}_{1} \mathrm{~s}$ in the main text. Alleles fixed by parent populations were more more dominant than recessive (mean $h_{\mathrm{z}}=0.64, \mathrm{SD}=0.079$ ), in agreement with Haldane's sieve.

The most readily apparent consequence of dominance with respect to hybrid fitness is that the availability of dominant alleles for adaptation affected hybrid fitness in a manner that depended on the form of natural selection (i.e., $\theta$; Fig. 2). Dominance tended to reduce hybrid fitness when selection was parallel, and had a less negative effect on hybrid fit- ness as selection became more divergent (i.e., as $\theta$ increased). Some effects depend on parameter values such as the selection strength and dimensionality, which we discuss where relevant in the main text and show fully in the supplement (Fig. S2) .

Parallel selection. Under completely parallel selection (i.e., $\theta=0^{\circ}$ ), we found that populations that adapted via alleles with variable dominance produced $\mathrm{F}_{1}$ hybrids with lower fitness than those using only additive alleles (Fig. 2A \& C). Dominant alleles reduce hybrid fitness under parallel selection because they cause hybrids to overshoot the phenotypic optimum (Fig. 2D \& E). Intuitively, expressing many partially dominant alleles in an entirely heterozygous $F_{1}$ causes hybrids to have trait values that are 'too large' for the environment and thus hybrids are less fit than parents with respect to their common phenotypic optimum. Supporting this, hybrid fitness decreased (for $\theta=0^{\circ}$ ) as the mean dominance coefficient of fixed mutations in the two parents increased (Fig. $3 \mathrm{~A})$. By contrast, the mean dominance coefficient had little effect on hybrid fitness at $\theta=180^{\circ}$ (see section on Divergent selection below). This result implies that the preferential fixation of dominant alleles (Haldane's [1924] Sieve) underlies the effect of dominance on hybrid fitness under parallel natural selection.

Allowing for parallel genetic evolution weakens, though does not eliminate, the negative effect of dominance on hybrid fitness at low angles (Fig. 2C). When parallel genetic evolution is possible, parents fix approximately $30 \%$ of alleles in common (Fig. S3). This genetic parallelism weakens the effect of dominance because hybrids are more homozygous and thus have a genotype and phenotype that is more similar to that of their parents (Fig. 2C-E). Similar to Thompson et al. (2019), we also observed that genetic parallelism rarely decreases to 0 even under complete divergent evolution where $\theta=180^{\circ}$ (Fig. S3). This indicates that populations fix some (nearly) neutral, compensatory, or deleterious alleles since the same mutant allele is unlikely to benefit both parental populations when $\theta=180^{\circ}$.

Divergent selection. Under completely divergent selection (i.e., $\theta=180^{\circ}$ ), we often observe a qualitatively different pattern than we did under parallel selection. In general, the effect of dominance on $F_{1}$ hybrid fitness is much less negative than under parallel selection. We found that dominant alleles can improve the fitness of hybrids under divergent selection compared to simulations with no dominance. This was counter to our expectations because we expected dominance to largely balance out so that hybrids would be phenotypically intermediate and equally distant to either phenotypic optima. Examining the phenotypes of hybrids revealed that dominance often caused hybrids to be closer to one of the parental phenotypic optima than the other, whereas adaptation without dominance yielded in relatively intermediate hybrids (Fig. 2D \& Fig. 2E; right side).

We reasoned that this pattern of dominance improving hybrid fitness is likely caused by sampling error-when few alleles are used for adaptation in each parental population, 
Relative $F_{1}$ fitness with and without dominance

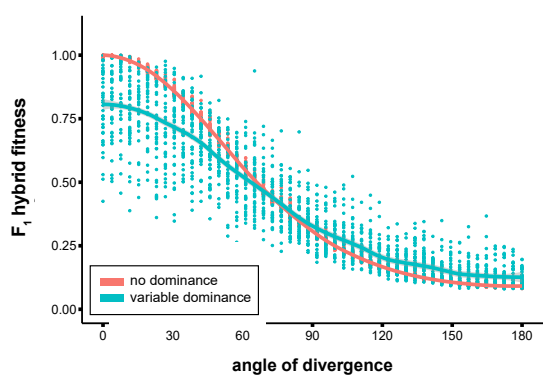

Relative $F_{1}$ fitness with and without dominance under genetic parallelism

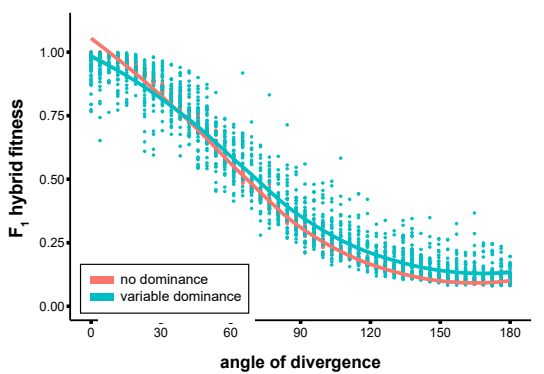

The effect of dominance on hybrid fitness across selection continuum

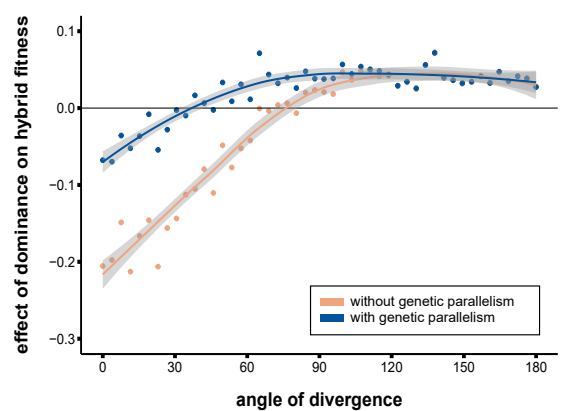

Phenotype ellipses in parallel and divergent evolution without genetic parallelism

Phenotype ellipses in parallel and divergent evolution with genetic parallelism
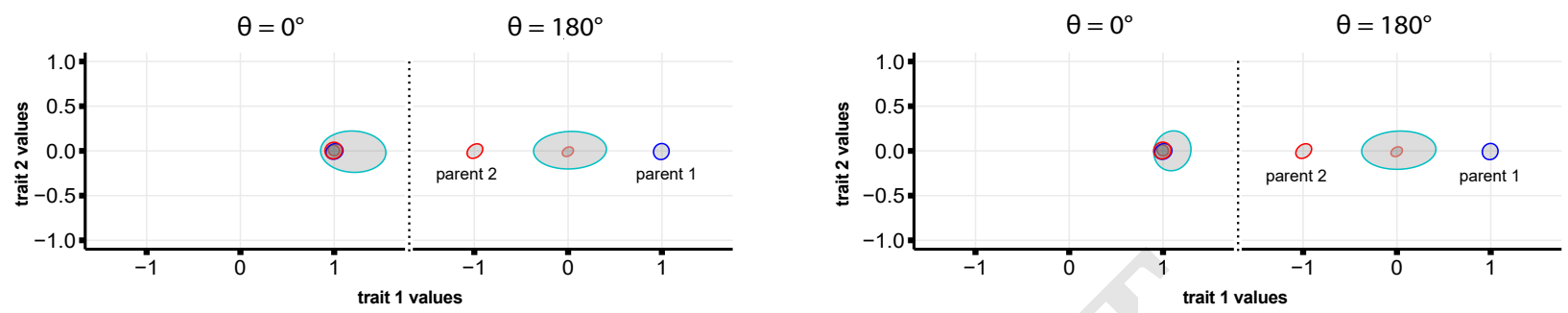

Fig. 2. How dominance affects hybrid fitness across the selection continuum. Panel (A) shows hybrid fitness values under additive and variable dominance adaptation conditions. Under parallel selection $\left(\theta=0^{\circ}\right)$ hybrids with no dominance have higher fitness than hybrids with variable dominance. As selection tends toward divergent $\left(\theta=180^{\circ}\right)$, variable dominance has a less negative effect on fitness. Results from 48 angles of divergence ( 40 replicates each) are plotted. Panel (B) shows the same relationships as in (A) under genetic parallelism. Genetic parallelism prevents the overshooting of the hybrids with dominance (blue line) under parallel selection. Panel (C) shows the effect of dominance on the fitness of $F_{1} s$ with and without parallel evolution, indicated by navy and orange curves, respectively. Each data point was computed by subtracting hybrid fitness under variable dominance from hybrid fitness under no dominance in Panels $(A)$ and $(B)$, then taking the mean of differences. Panels (D) and (E) show $F_{1}$ hybrid and parental phenotypes without and with genetic parallelism, respectively, with each replicate simulation contributing one value and ellipses are drawn across replicates. Dark red and blue ellipses are centred on the parental optima. Hybrid phenotype ellipses with and without dominance are shown in turquoise and orange colours. Dominance causes hybrids to overshoot the optimum at $[1,0]$ under parallel selection $\left(\theta=0^{\circ}\right)$, but brings them closer to one of the two phenotypic optima under divergent selection $\left(\theta=180^{\circ}\right)$.

the mean dominance coefficient of alleles fixed during adaptation could differ markedly between them. Supporting this, we found that as the difference in mean $h_{z}$ between $\mathrm{P}_{\mathrm{A}}$ and $\mathrm{P}_{\mathrm{B}}$ - the 'dominance imbalance' (Eqn. 5)-increases, the mean fitness of hybrids improves (Fig. 3B); dominance imbalance does not strongly affect fitness under parallel evolution. Thus, chance fixation of alleles with different dominance underlie the fitness effects of dominant alleles under divergent selection.

We further conducted simulations with a variety of mutational effect size values (i.e., $\alpha$ ) with the expectation that aforementioned sampling error would be greatest for larger values of $\alpha$ because fewer mutations would be acquired during adaptation. Likewise, we expected to see less variation between different simulation runs for smaller values of $\alpha$ because this would lead to accumulation of more mutations which in return, would lead to a greater variability of dominance coefficients. The results support this hypothesis: dominance has a diminishing effect on $\mathrm{F}_{1}$ fitness when mutational effect sizes $(\alpha)$ are small, regardless of the availability of genetic parallelism (Fig. 3C). With decreasing mutational effect sizes, parental populations fix more mutations during adaptation, decreasing the sampling error that causes the dominance imbalance (Fig. 3D). Previous theoretical studies showed that reproductive isolation establishes in populations more rapidly when mutations with large effect sizes drive adaptation, in both haploid and diploid models (Barton 2001). Our results suggest that adding dominance strengthens this conclusion under parallel selection, but can weaken it under increasingly divergent selection because hybrid fitness improves.

\section{Effect of dominance on $\mathrm{F}_{\mathbf{2}}$ and $\mathrm{BC}_{\mathbf{1}}$ hybrid fitness. As} with $F_{1}$ hybrids, dominance has the most negative impact on hybrid fitness under parallel selection and its effect becomes less negative as $\theta$ increases. (Fig. S4). The effects of dominance are most pronounced in the $\mathrm{F}_{1}$ because $\mathrm{BC}_{1} \mathrm{~s}$ and $\mathrm{F}_{2} \mathrm{~S}$ are half as heterozygous on average and therefore dominance affects the phenotype less. The effect of genetic parallelism is similar to the $F_{1}$ case as well-ameliorating the negative effects of dominance under parallel selection and having little effect under divergent selection. See Fig. S4 for showing raw values for $\mathrm{BC}_{1}$ and $\mathrm{F}_{2}$ hybrid fitness under both parallel evolution scenarios, see Fig. S5 for a figure comparing fitness and and the dominance-effect on fitness among hybrid classes directly, and see Fig. S6 for phenotype distributions for $\mathrm{BC}_{1}$ and $\mathrm{F}_{2}$ hybrids.

Effects of alternate parameter values. To assess the robustness of our findings, we ran simulations with various dimensionality $(n)$, distance to optima $(d)$ and selection 
bioRxiv preprint doi: https://doi.org/10.1101/2021.06.30.450598; this version posted July 1, 2021. The copyright holder for this preprint (which was not certified by peer review) is the author/funder, who has granted bioRxiv a license to display the preprint in perpetuity. It is made available under ACC-BY-NC-ND 4.0 International license.

A Hybrid fitness across average dominance coefficient values

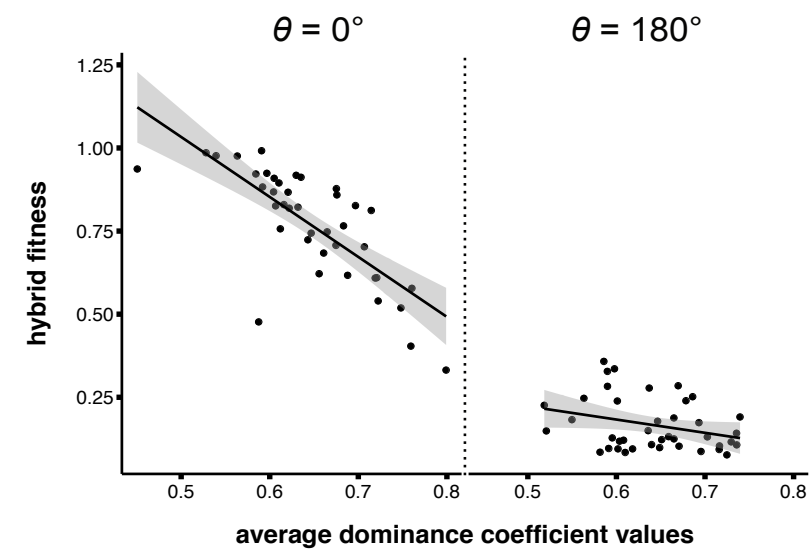

Effect of dominance on hybrid fitness across mutation effect sizes

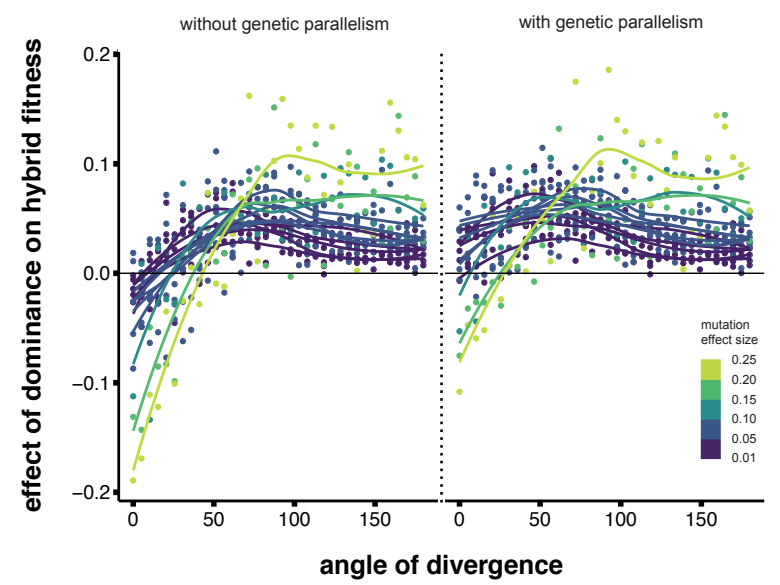

B Hybrid fitness across dominance imbalance values

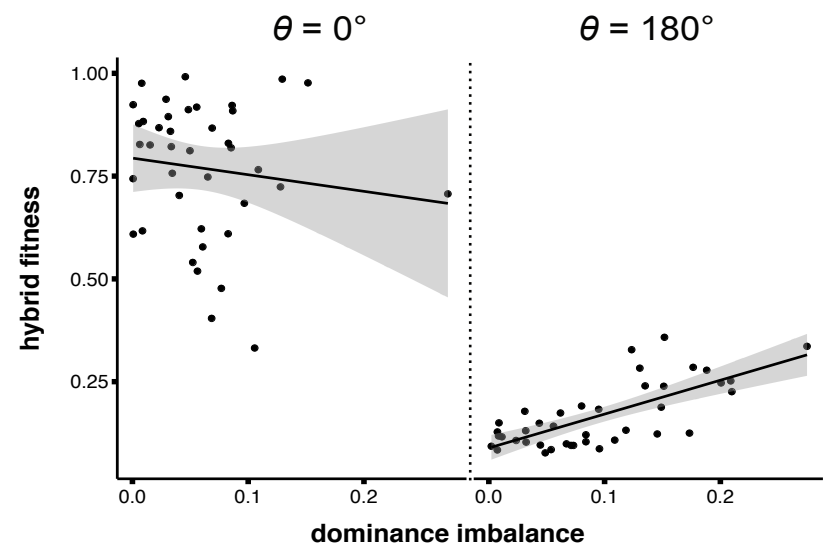

D Dominance imbalance across mutational effect sizes

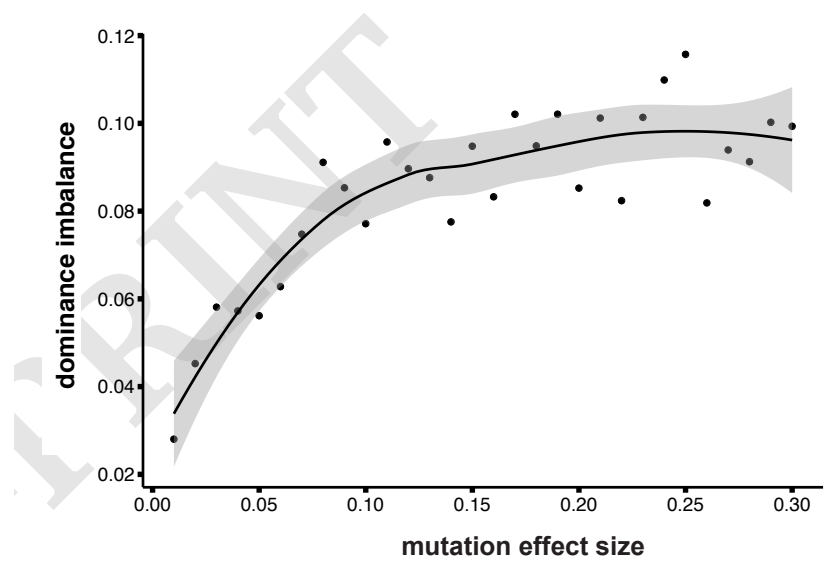

Fig. 3. Causes of the effect of dominance on $F_{1}$ hybrid fitness. Panel (A) shows how $F_{1}$ hybrid fitness depends on the mean dominance coefficient (i.e., $h_{z}$ ) values of fixed mutations across both parental populations. Greater mean dominance decreases hybrid fitness under parallel selection but has relatively little effect under divergent selection. Panel (B) illustrates the relationship between hybrid fitness and average dominance imbalance (Eqn. 5). Dominance imbalance increases hybrid fitness under divergent selection but has little effect under parallel selection. Panel (C) shows how the effect of dominance on hybrid fitness changes across various values of mutation effect size (i.e., $\alpha$ ) without (left) and with (right) genetic parallelism. With higher $\alpha$ values, the effect of dominance on hybrid fitness (difference between dominance and no dominance scenarios) diminishes. Panel (D) shows the increase in the average dominance imbalance as a result of higher mutational effect sizes.

strength $(\sigma)$ values.

We first evaluated the effects of dimensionality (i.e., $n$ ) from $n=2-7$. As dimensionality increases, the mean dominance coefficients of alleles fixed by parental (and the dominance imbalance) populations decreases substantially (Fig. S7A \& B). We also find that the mean effect size of fixed mutations decreases slightly as dimensionality increases (Fig. S7C). The general pattern of dominance having an increasingly positive effect on hybrid fitness as $\theta$ increases (as in Fig. 2C) is robust to variation in $n$. However, dominance becomes more deleterious as $n$ increases, which we suspected is because deleterious the pleiotropic effects of mutations are amplified by additional dimensions since there are more ways for them to 'go wrong' (Orr, 2000). Supporting this hypothesis, we find that the extent to which dominance causes hybrids to express maladaptive transgressive phenotype in additional dimensions increases with $n$ (Fig. S7D). Clearly, this transgression eventually outweighs the beneficial effects of the dominance imbalance that predominates at lower dimensionalities (Fig. 3).

We also observe that the main patterns we identified at $d=1$ and $\sigma=5$ are robust to alternative values of $d$ and $\sigma$. Specifically, dominance affects $\mathrm{F}_{1}$ hybrid fitness in a manner dependent on $\theta$ (Fig. S2B), and genetic parallelism diminishes negative effects of dominance at lower angles of divergence by preventing overshooting. With higher $d$, the effect of dominance on $F_{1}$ fitness becomes increasingly negative at low angles because more mutations fix when the optimum is further and thus we observe greater overshooting. Likewise with higher $\sigma$ leads to an increasingly deleterious effect of dominance on hybrid fitness (Fig. S2C) because the fitness cost of overshooting the optimum is greater. For large angles, the effect of dominance weakens as $d$ increases. This is likely because more mutations fix thereby decreasing the 
dominance imbalance, and hybrids are in a deep fitness valley and small deviations cause them to make little progress toward reaching parental optima.

Implications of dominance for speciation. Using simulations based on Fisher's (1930) geometric model, we evaluated how dominance affects progress toward speciation via extrinsic hybrid fitness. Under nearly all explored parameter combinations, we found that dominance reduces hybrid fitness under parallel selection due to the transgressive phenotypes expressed by the $F_{1}$ hybrids (i.e., 'overshooting'). Because hybrids express trait values that depart from the range seen in parents, they have low fitness in the parental environment (Barton, 2001; Schneemann et al., 2020; Thompson et al., 2019; Yamaguchi and Otto, 2020). Previous empirical studies found that transgressive segregation is common. Rieseberg et al. (1999) identified transgressive segregation in 110 of 113 studies on plant species and 45 of 58 animal species. A widely accepted view is that in a purely additive genetic background, transgressive hybrid phenotypes can only be observed in $F_{2}$ hybrids due to the recombination of alleles at the quantitative trait loci (QTL). Stelkens and Seehausen (2009) and Stelkens et al. (2009) predicted that transgression can also occur in $F_{1}$ hybrids under dominance or epistasis, and found across 62 plant and 15 animal studies that the frequency of transgressive traits increases with increasing phenotypic similarity between the parents, showing that the outcome of hybridization is predictable from the genetic variation between the parents. Our study provides theoretical support for the hypothesis that transgressive segregation readily occurs given parallel natural selection and some variation in the dominance of alleles used for adaptation. Importantly, the effect of dominance under a parallel selection regime was attenuated by parallelism at the genetic level, whereas its effect under divergent selection was not. Thus, dominance should be expected to facilitate progress toward speciation by parallel natural selection.

The effect of dominance on hybrid fitness generally became less negative as the angle between optima (i.e., $\theta$ ) increased. When dimensionality was low, dominance typically increased hybrid fitness under divergent selection because it caused hybrids to be considerably closer to one optimum than the other. This implies that, for cases where dimensionality is low, dominance will typically work against speciation by divergent natural selection (Nosil, 2012; Schluter, 1996, 2001). A previous synthetic study of $F_{1}$ hybrid phenotype expression found that $F_{1} s$ are often more similar to one parent than the other ('parent-bias') as a result of dominance (Thompson et al., 2021), which is consistent with the patterns documented herein. However, dominance reduced hybrid fitness under divergent selection when dimensionality was high. This is largely because it caused hybrids to exhibit transgressive segregation for traits under stabilizing selection which is the main mode of speciation considered by Barton (2001) and Thompson (2020). This transgressive segregation is equivalent to 'trait mismatch', which is common in hybrids (Thompson et al., 2021). As dimensionality increases, increasing mismatch overrides parent-bias and causes hybrid fitness to decline (i.e., $n$ ). Thus, for complex organisms, dominance can be expected to facilitate progress toward speciation via divergent selection because it results in trangressive segregation and trait mismatch.

In this study we made several assumptions that affect our conclusions. First, we have assumed that adaptation happens in allopatry, and it would be useful to determine if gene flow affects patterns beyond simple allele sharing (which we consider via altering our assumption of genetic parallelism). Second, we only consider early generation hybrids, and it would be useful to extend this framework to ongoing hybridization in a hybrid zone (Irwin, 2020). Finally, we did not explore modularity (i.e., restricted pleiotropy; Yamaguchi and Otto 2020) which could ameliorate the effects of dimensionality. Nevertheless, our findings are consistent with several empirical studies and provide novel theoretical insight into the role that a fundamental aspect of genetic architecture-the dominance of quantitative trait loci-plays in speciation.

\section{Data accessibility}

All code necessary to repeat the procedure outlined in this study is available at https://github.com/amunzur/SpeciationWithDominance.

To rerun the code, we recommend you to create a conda environment using the yaml file provided.

\section{Acknowledgements}

Feedback from S. Blain, M. Osmond, S. Otto, and D. Schluter benefited the manuscript. R. Henriques created the bioR $\chi$ iv IATEXtemplate. ADM was supported by a UBC International Work Learn Award, and Karen McKellin International Leader of Tomorrow Award. KAT was supported by an NSERC Canada Graduate Scholarship (CGS-D), a UBC Four Year Fellowship, a Killam Doctoral Scholarship, and a British Columbia Graduate Scholarship.

\section{Author contributions}

ADM and KAT designed the study. ADM developed the computer simulations in consultation with KAT. ADM analyzed the data and produced the figures. ADM and KAT wrote the manuscript.

\section{Bibliography}

\footnotetext{
Adams, D. C. and Collyer, M. L. (2009). A general framework for the analysis of phenotypic trajectories in evolutionary studies. Evolution, 63(5):1143-1154.

Barton, N. H. (2001). The role of hybridization in evolution. Molecular Ecology, 10(3):551-568.

Bolnick, D. I., Barrett, R. D. H., Oke, K. B., Rennison, D. J., and Stuart, Y. E. (2018). (Non)Parallel Evolution. Annual Review of Ecology, Evolution, and Systematics, 49:303-330.

Chevin, L.-M., Decorzent, G., and Lenormand, T. (2014). Niche dimensionality and the genetics of ecological speciation. Evolution, 68(5):1244-1256.

Conte, G. L., Arnegard, M. E., Peichel, C. L., and Schluter, D. (2012). The probability of genetic parallelism and convergence in natural populations. Proceedings of the Royal Society $B$ : Biological Sciences, 279(1749):5039-5047.

Coyne, J. A. and Orr, H. A. (2004). Speciation. Sinauer.

Crow, J. F. and Kimura, M. (1970). An introduction to population genetics theory. Harper and Row, New York.

De Lisle, S. P. and Bolnick, D. I. (2020). A multivariate view of parallel evolution. Evolution, 74(7):1466-1481
} 
bioRxiv preprint doi: https://doi.org/10.1101/2021.06.30.450598; this version posted July 1, 2021. The copyright holder for this preprint (which was not certified by peer review) is the author/funder, who has granted bioRxiv a license to display the preprint in perpetuity. It is made available under aCC-BY-NC-ND 4.0 International license.

Fisher, R. A. (1930). The Genetical Theory of Natural Selection. Oxford University Press, Oxford, UK.

Haldane, J. B. S. (1924). A mathematical theory of natural and artificial selection, Part I. Transactions of the Cambridge Philosophical Society, 23:19-41.

Haldane, J. B. S. (1927). A mathematical theory of natural and artificial selection, Part V: selection and mutation. Mathematical Proceedings of the Cambridge Philosophical Society, 23(7):838844.

Irwin, D. E. (2020). Assortative mating in hybrid zones is remarkably ineffective in promoting speciation. The American Naturalist, 195(6):150-167.

James, M. E., Wilkinson, M. J., North, H. L., Engelstädter, J., and Ortiz-Barrientos, D. (2020). A framework to quantify phenotypic and genotypic parallel evolution. bioRxiv.

Kimura, M. (1965). A stochastic model concerning the maintenance of genetic variability in quantitative characters. Proceedings of the National Academy of Sciences of the United States of America, 54(3):731-736.

Kimura, M. (1969). The number of heterozygous nucleotide sites maintained in a finite population due to steady flux of mutations. Genetics, 61(4):893-903.

Lande, R. (1979). Quantitative genetic analysis of multivariate evolution, applied to brain: body size allometry. Evolution, 33(1):402-416.

Langerhans, R. B. and Riesch, R. (2013). Speciation by selection: A framework for understanding ecology's role in speciation. Current Zoology, 59(1):31-52.

Miller, C. T., Glazer, A. M., Summers, B. R., Blackman, B. K., Norman, A. R., Shapiro, M. D., Cole, B. L., Peichel, C. L., Schluter, D., and Kingsley, D. M. (2014). Modular skeletal evolution in sticklebacks is controlled by additive and clustered quantitative trait loci. Genetics, 197(1):405-420.

Nosil, P. (2012). Ecological speciation. Oxford University Press, Oxford, UK.

Oke, K. B., Rolshausen, G., LeBlond, C., and Hendry, A. P. (2017). How parallel is parallel evolution? A comparative analysis in fishes. The American Naturalist, 190(1):1-16.

Ono, J., Gerstein, A. C., and Otto, S. P. (2017). Widespread genetic incompatibilities between firststep mutations during parallel adaptation of $<i>$ Saccharomyces cerevisiae $</ i>$ to a common environment. PLoS Biology, 15(1):e1002591.

Orr, H. A. (2000). Adaptation and the cost of complexity. Evolution, 54(1):13-20.

Rabosky, D. L., Chang, J., Title, P. O., Cowman, P. F., Sallan, L., Friedman, M., Kaschner, K. Garilao, C., Near, T. J., Coll, M., and Alfaro, M. E. (2018). An inverse latitudinal gradient in speciation rate for marine fishes. Nature, 559(7714):392-395.

Rieseberg, L. H., Archer, M. a., and Wayne, R. K. (1999). Transgressive segregation, adaptation and speciation. Heredity, 83:363-372.

Schluter, D. (1996). Ecological speciation in postglacial fishes. Philosophical Transactions of the Royal Society B: Biological Sciences, 351(1341):807-814.

Schluter, D. (2000). The Ecology of Adaptive Radiation. Oxford University Press, New York.

Schluter, D. (2001). Ecology and the origin of species. Trends in Ecology and Evolution, 16(7):372-380

Schluter, D. (2009). Evidence for ecological speciation and its alternative. Science, 323(2008):737-741.

Schneemann, H., De Sanctis, B., Roze, D., Bierne, N., and Welch, J. J. (2020). The geometry and genetics of hybridization. Evolution, 74(12):2575-2590.

Stelkens, R. and Seehausen, O. (2009). Genetic distance between species predicts novel trait expression in their hybrids. Evolution, 63(4):884-897.

Stelkens, R. B., Schmid, C., Selz, O., and Seehausen, O. (2009). Phenotypic novelty in experimental hybrids is predicted by the genetic distance between species of cichlid fish. BMC Evolutionary Biology, 9:283.

Stuart, Y. E., Veen, T., Weber, J. N., Hanson, D., Ravinet, M., Lohman, B. K., Thompson, C. J., Tasneem, T., Doggett, A., Izen, R., Ahmed, N., Barrett, R. D. H., Hendry, A. P., Peichel, C. L., and Bolnick, D. I. (2017). Contrasting effects of environment and genetics generate a continuum of parallel evolution. Nature Ecology \& Evolution, 1(6):0158.

Tenaillon, O. (2014). The utility of Fisher's geometric model in evolutionary genetics. Annual Review of Ecology, Evolution, and Systematics, 45(1):179-201.

Thompson, K. A. (2020). Experimental hybridization studies suggest that pleiotropic alleles commonly underlie adaptive divergence between natural populations. American Naturalist, 196(1):E16-E22.

Thompson, K. A., Osmond, M. M., and Schluter, D. (2019). Parallel genetic evolution and speciation from standing variation. Evolution Letters, 3(2):129-141.

Thompson, K. A., Urquhart-Cronish, M., Whitney, K. D., Rieseberg, L. H., and Schluter, D. (2021) Patterns, predictors, and consequences of dominance in hybrids. The American Naturalist, In press.

Yamaguchi, R. and Otto, S. P. (2020). Insights from Fisher's geometric model on the likelihood of speciation under different histories of environmental change. Evolution, 74(8):1603-1619. 


\section{Supplementary material 1: Supplementary Methods}

To determine the fixation probability of a mutation given its phenotypic effect, we used main text Eqn. 4 . We compared the result of this equation to two others, shown below:

$$
\pi \equiv \frac{1-e^{-4 s h_{w} N}}{1-e^{-4 s h_{w} N}}
$$

where $p$, the initial frequency of the mutation, is $\frac{1}{2 N}$, assuming it arises as a single copy in a diploid population, and $s$ stands for the selection coefficient of the mutation, calculated by Equation 2 in the main text. The equation above, first derived by Kimura $(1965 ; 1969)$, assumes a large and constant population size with random mating. We also used another equation by Crow and Kimura (1970) where $p=\frac{1}{2 N}$ and $h_{w}$ stands for the dominance for fitness of the mutation:

$$
\pi \equiv \frac{\int_{0}^{p} e^{2 N s\left[\left(2 h_{w}-1\right) x(1-x)-2 N s x\right]} \mathrm{d} x}{\int_{0}^{1} e^{2 N s\left[\left(2 h_{w}-1\right) x(1-x)-2 N s x\right]} \mathrm{d} x}
$$

In both Equation S.1 and Equation S.2, $N$ stands for population size, which we set to $N=1000$. We tested the fixation probability of a 1000 randomly generated mutations using Equation 4, Equation S.1 and Equation S.2. The linear regression plots with $R^{2}$ information is given in Fig. S1 where fixation probability of a given mutation calculated by either one of the three equation is comparable. We used Eqn. 4 in the main text since it is the simplest of equations that all gave similar results and outcomes. 
bioRxiv preprint doi: https://doi.org/10.1101/2021.06.30.450598; this version posted July 1, 2021. The copyright holder for this preprint (which was not certified by peer review) is the author/funder, who has granted bioRxiv a license to display the preprint in perpetuity. It is made available under aCC-BY-NC-ND 4.0 International license.

\section{Supplementary material 2: Supplementary figures}

A Fixation probabilities computed by (4) and (S.1)

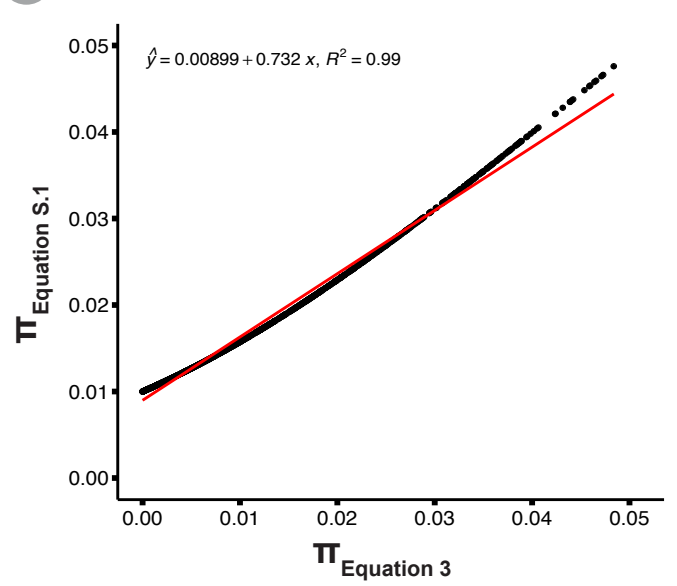

B Fixation probabilities computed by (4) and (S.2)

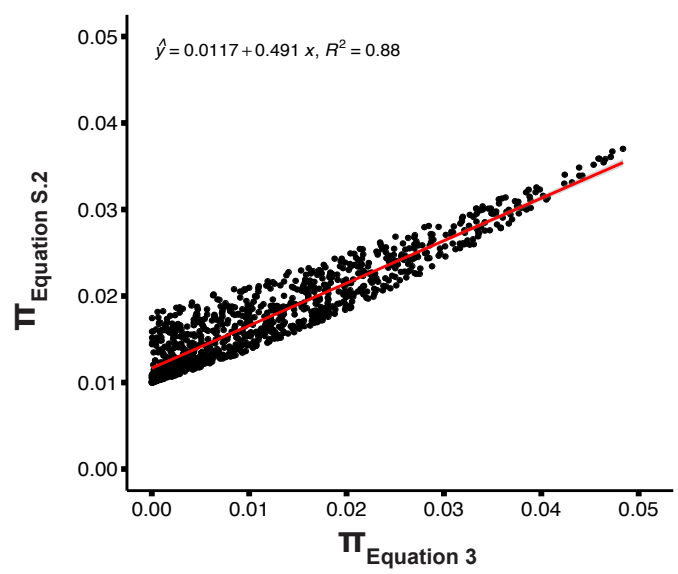

Fig. S1. Linear regression between alternative equations for calculating the probability of fixation ( $\pi$ ) of a mutation. Panel (A) shows the relationship between Eqn. 4 and Eqn. S.1. Panel (B) shows linear regression between Eqn. 4 and Eqn. S.2. Red lines are $1: 1$ lines. 
bioRxiv preprint doi: https://doi.org/10.1101/2021 06.30.450598; this version posted July 1, 2021. The copyright holder for this preprint (which was not certified by peer review) is the author/funder, who has granted bioRxiv a license to display the preprint in perpetuity. It is made available under aCC-BY-NC-ND 4.0 International license.

A Effect of dominance on $F_{1}$ fitness under various dimensionality values $(n)$

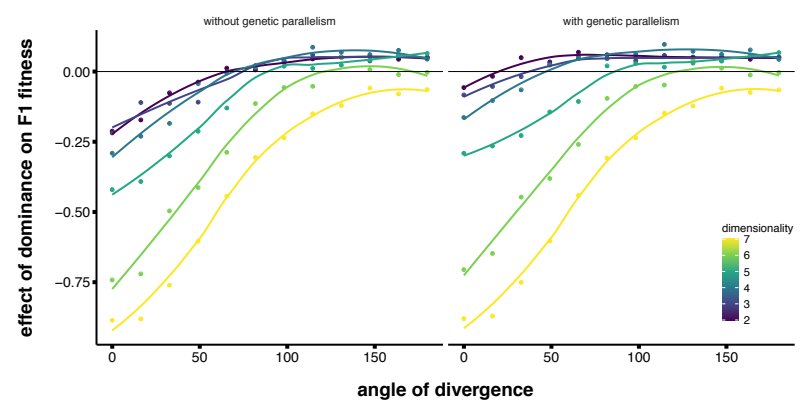

B Effect of dominance on $\mathrm{F}_{1}$ fitness under various distance to optima values $(d)$

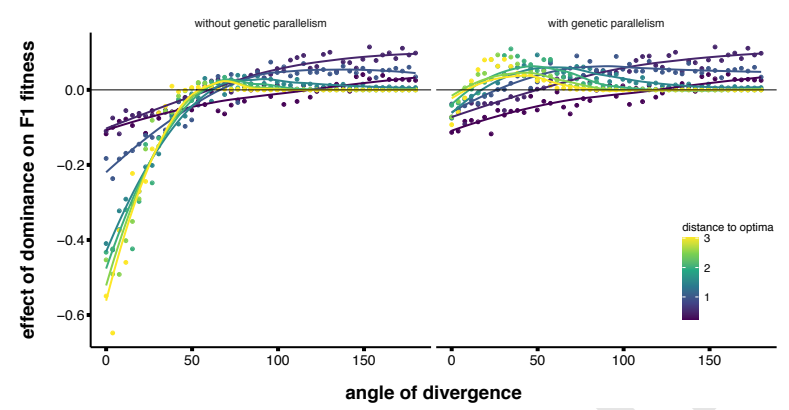

Effect of dominance on $F_{1}$ fitness under various selection strengths $(\sigma)$

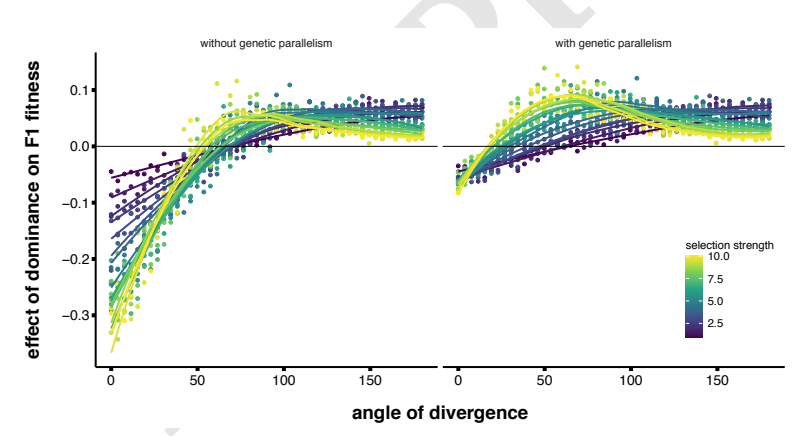

Fig. S2. The effect of dominance on $F_{1}$ fitness with changing dimensionality $(n)$, distance to optima (d) and selection strength $(\sigma)$ values. This figure is adapted from Fig. $3 \mathrm{C}$ where the effect of dominance with and without genetic parallelism is plotted under various parameters. 


\section{Genetic parallelism across the selection continuum}

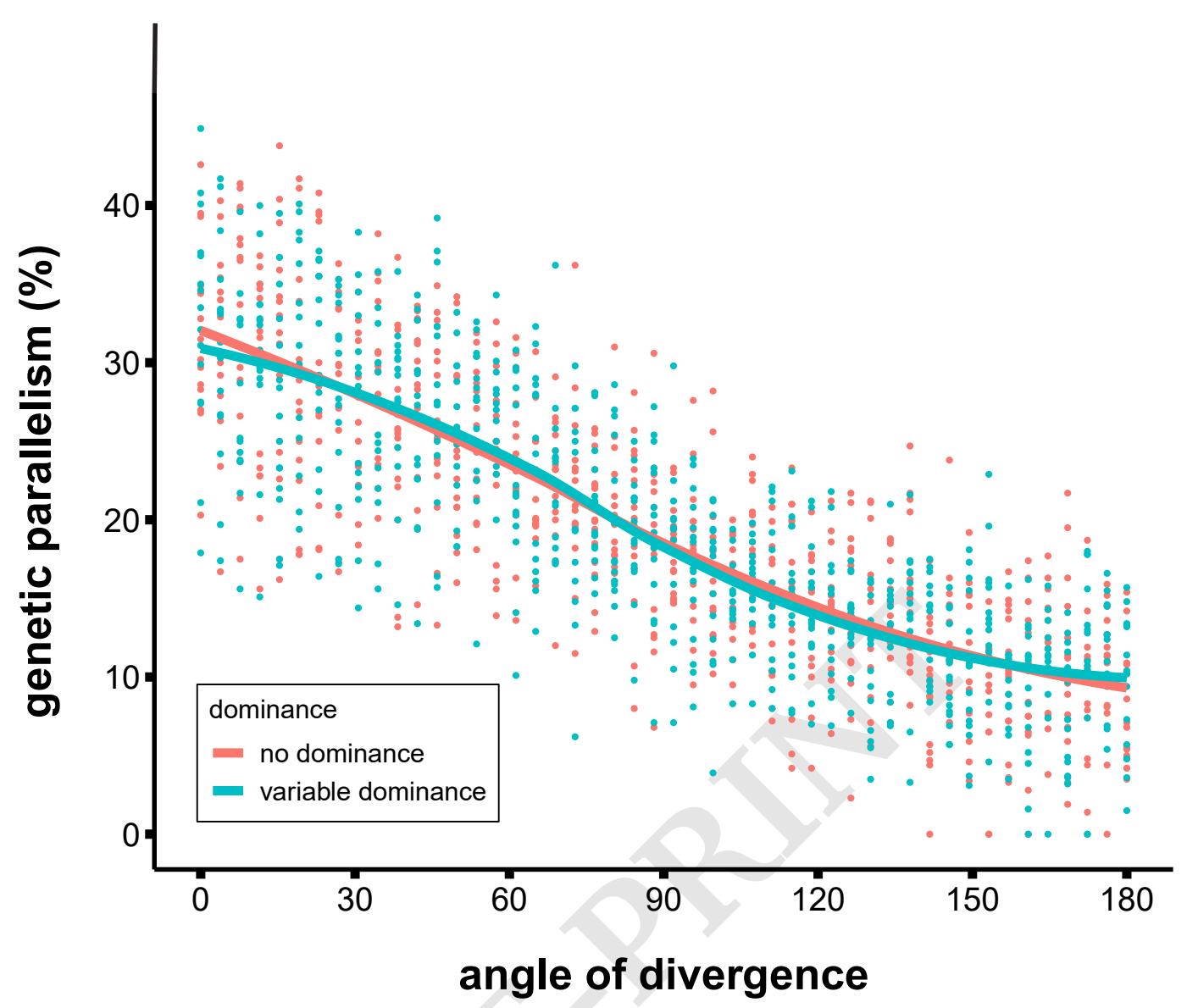

Fig. S3. Genetic parallelism across the selection continuum with and without genetic dominance. Genetic parallelism, the percentage of shared alleles between the parental populations, decreases as the angle of divergence increases. In these simulations, 48 angles and 15 replicates have been used. The horizontal line at $y=0$ indicates net effect of 0 on hybrid fitness. 
bioRxiv preprint doi: https://doi.org/10.1101/2021.06.30.450598; this version posted July 1, 2021. The copyright holder for this preprint (which was not certified by peer review) is the author/funder, who has granted bioRxiv a license to display the preprint in perpetuity. It is made available under aCC-BY-NC-ND 4.0 International license.
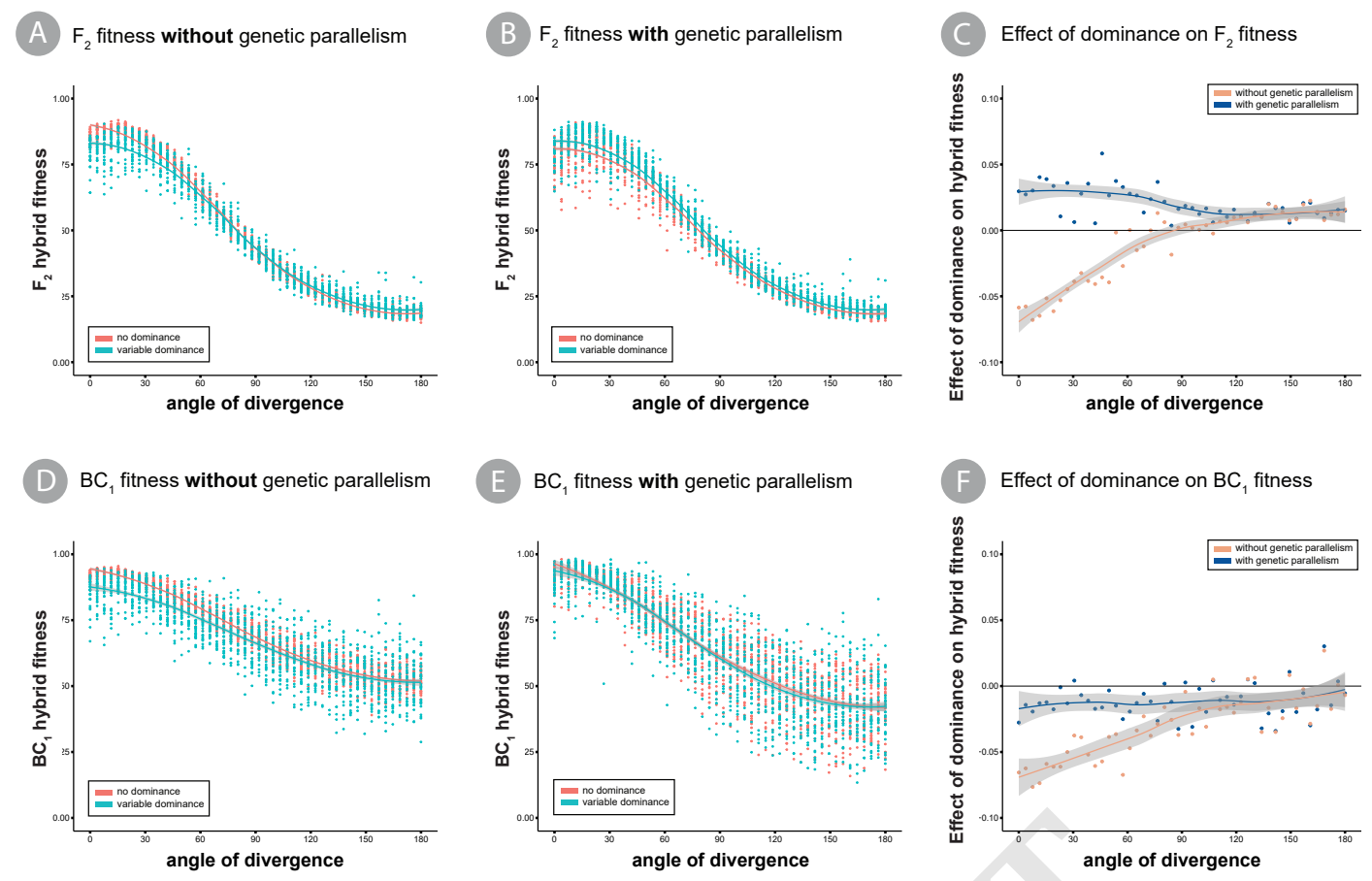

Fig. S4. $F_{2}$ and $B_{1} C_{1}$ hybrid fitness. Panels $(A)$ and $(B)$ show $F_{2}$ hybrid fitness without and with genetic parallelism. Panel (C) shows the effect of dominance on $\mathrm{F}_{2}$ fitness, similar to Fig. $2 \mathrm{C}$. Panels $(\mathrm{D})$ and $(\mathrm{E})$ show $\mathrm{BC}_{1}$ hybrid fitness without and with genetic parallelism. Panel $(F)$ shows the effect of dominance on $\mathrm{BC}_{1}$ fitness. 


\title{
The effect of dominance on hybrid fitness
}

\author{
without genetic parallelism \\ with genetic parallelism
}

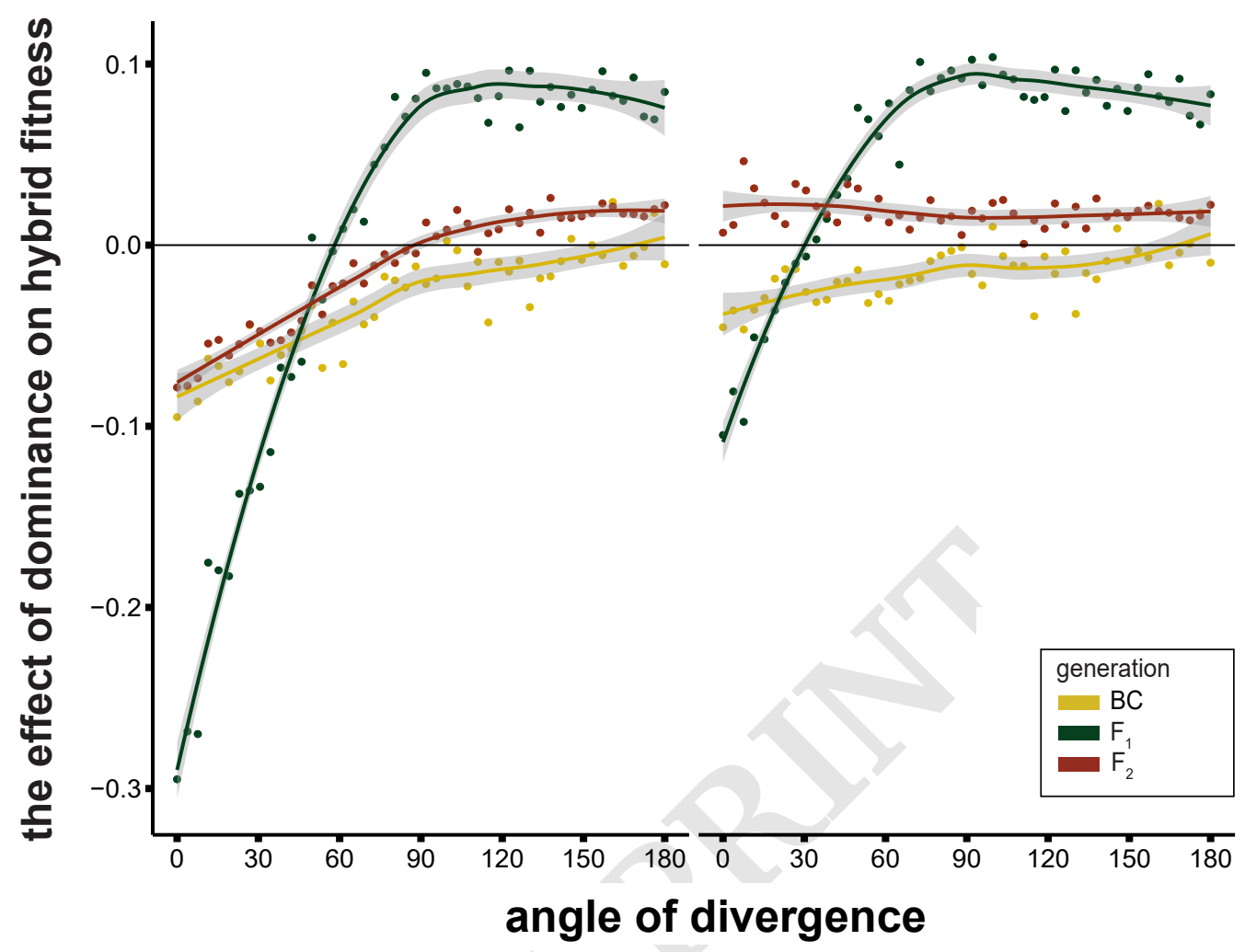

Fig. S5. The effect of dominance on the $F_{1}$ fitness across the selection continuum without (left) and with (right) genetic parallelism. The effect of dominance was calculated by subtracting hybrid fitness without dominance from hybrid fitness with dominance. The horizontal line at $y=0$ indicates net effect of 0 on hybrid fitness. 
bioRxiv preprint doi: https://doi.org/10.1101/2021.06.30.450598; this version posted July 1, 2021. The copyright holder for this preprint (which was not certified by peer review) is the author/funder, who has granted bioRxiv a license to display the preprint in perpetuity. It is made available under aCC-BY-NC-ND 4.0 International license.

$F_{2}$ phenotypes under additive and variable dominance when $\theta=0^{\circ}$
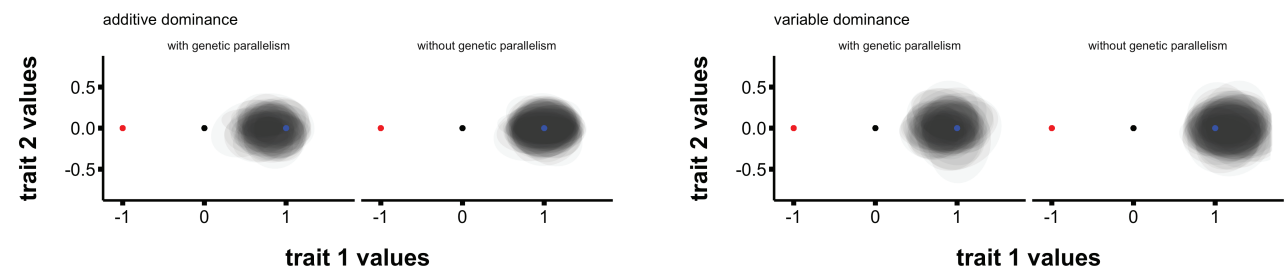

$F_{2}$ phenotypes under additive and variable dominance when $\theta=180^{\circ}$
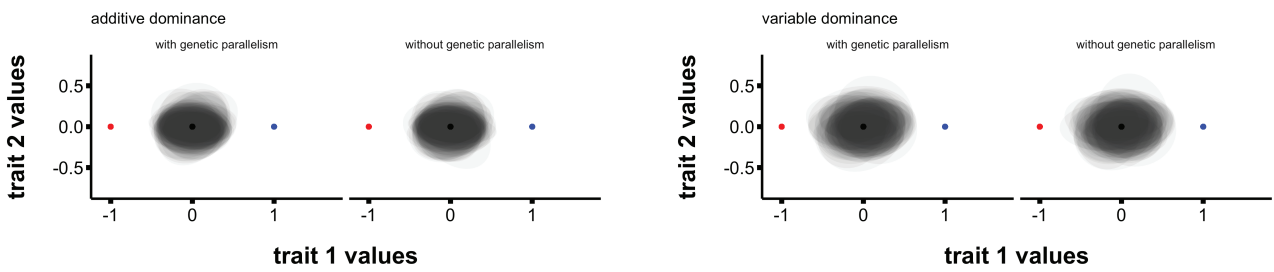

$\mathrm{BC}_{1}$ phenotypes under additive and variable dominance when $\theta=0^{\circ}$
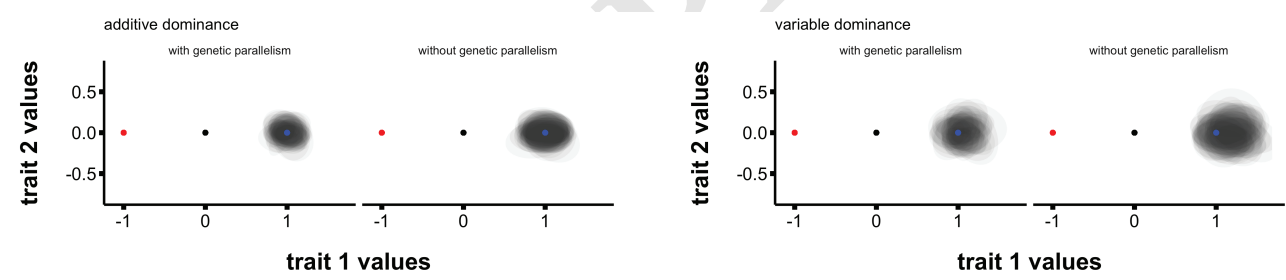

D $\mathrm{BC}_{1}$ phenotypes under additive and variable dominance when $\theta=180^{\circ}$
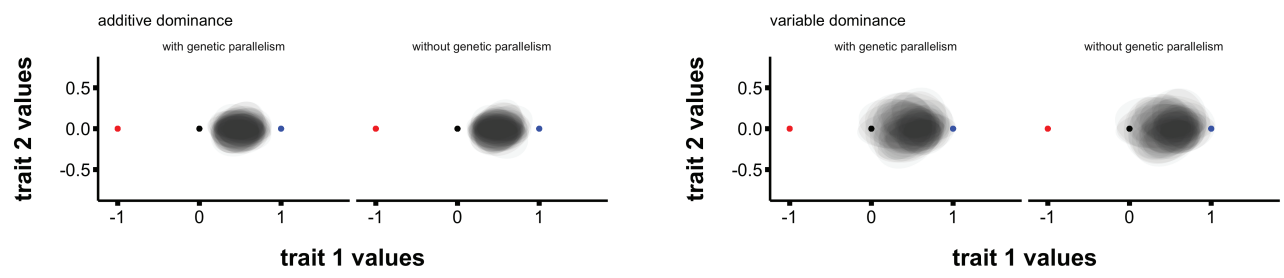

Fig. S6. $F_{2}$ and backcross phenotypes visualized as ellipses. Panels $(A)$ and $(B)$ show $F_{2}$ phenotypes with angles of divergence of $\theta=0^{\circ}$ and $\theta=180^{\circ}$. Panels (C) and (D) show $\mathrm{BC}_{1}$ phenotypes with angles of divergence of $\theta=0^{\circ}$ and $\theta=180^{\circ}$. All panels show the phenotype information with and without genetic parallelism under the indicated genetic architecture. Red and blue dots indicate the parental optima, and the black dot points at the origin where adaptation started from. All individuals have been plotted separately, darker shades indicate the regions where the majority of the hybrids are found. In a single simulation, $500 \mathrm{~F}_{2}$ and $500 \mathrm{BC}_{1}$ hybrid individuals have been made. 
bioRxiv preprint doi: https://doi.org/10.1101/2021.06.30.450598; this version posted July 1, 2021. The copyright holder for this preprint (which was not certified by peer review) is the author/funder, who has granted bioRxiv a license to display the preprint in perpetuity. It is made available under aCC-BY-NC-ND 4.0 International license.

A Average dominance coefficient values across dimensionality

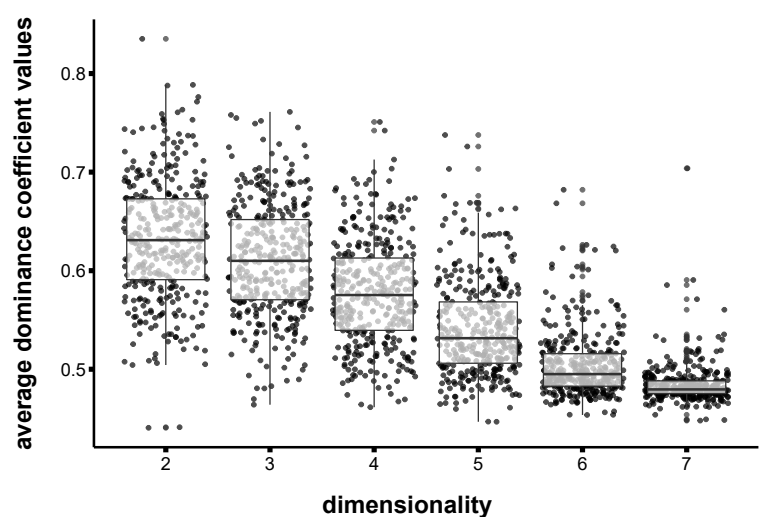

C Average mutational euclidian distance

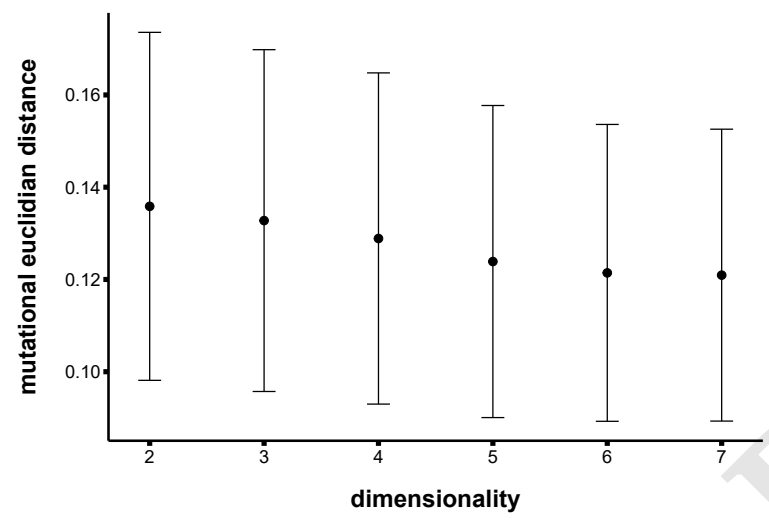

B Dominance imbalance across dimensionality

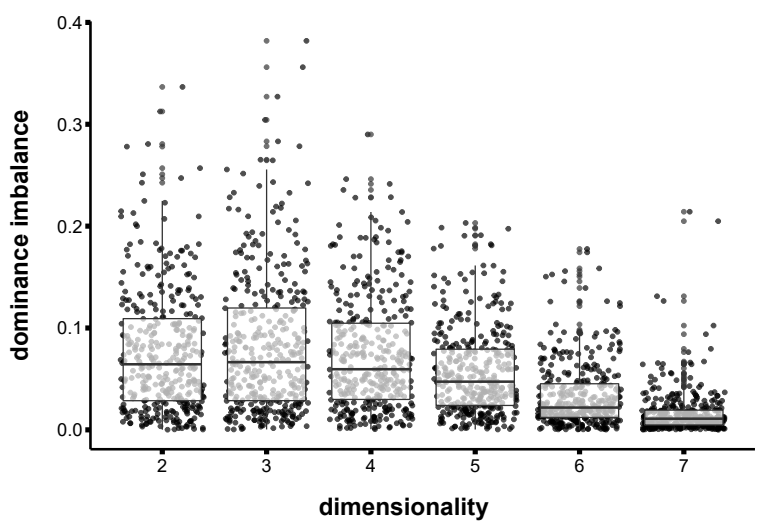

Dean trait values for $\theta=180^{\circ}$

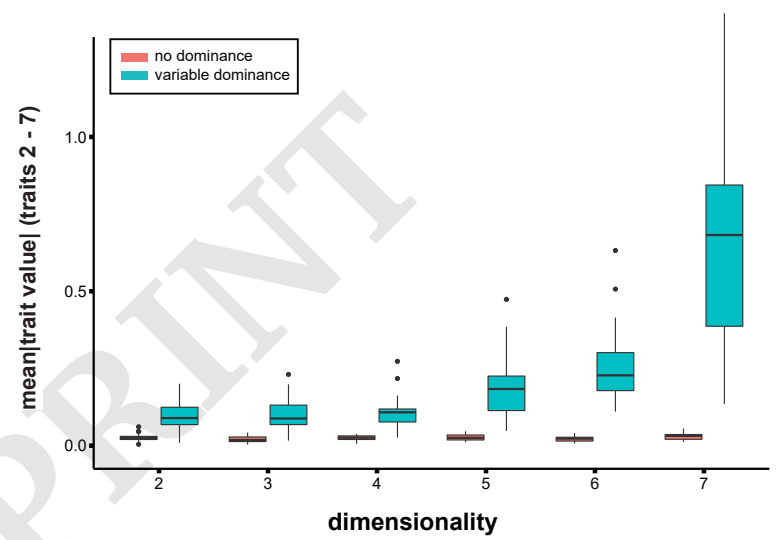

Fig. S7. Average dominance coefficients and dominance imbalance across dimensionality. Panel (A) shows the average dominance coefficient values of fixed mutations across dimensionality (varying number of traits). Panel (B) shows the dominance imbalance across dimensionality calculated according to Eqn. 5 was used to calculate dominance imbalance. Panel (C) shows the mean mutational vector sizes (euclidean distance) of fixed mutations during adaptation. Error bars represent half of the standard deviation of the dominance coefficients of fixed mutations. Panel (D) includes the summed trait values for different dimensionality cases under divergent selection $\left(\theta=180^{\circ}\right)$, calculated according to the formula $\sum_{2} 2^{n}\left|z_{n}\right|$. 